\title{
Reviving. Surviving. Thriving. The Homerton deteriorating patient programme
}

\author{
Authors: Billy Cheung, ${ }^{*}$ Grace Walker, ${ }^{*}$ Ian Donaldson, Kieran Palmer, Paul Tern and Carlo Prina
}

\section{Introduction}

Improving management of deterioration is a national priority. Despite this, measuring performance and improvements in deterioration can be challenging. Mortality and cardiac arrest statistics are often used as indicators but are limited in their value. The Trust leadership at Homerton, a 500-bed district general hospital, noted a pattern in incident reports around deteriorating inpatients. We examined our current response to deterioration and targeted areas to improve performance.

\section{Materials and methods}

\section{Data collection}

We collected data from a range of sources, both qualitative (thematic review of intensive treatment unit (ITU) admissions and staff feedback) and quantitative (escalation and response times).

> We performed thematic analysis of unplanned admissions to ITU December 2017-February $2018(n=51)$. We identified common themes.

$>$ Delay in referral to ITU.

> Delayed response to physiological deterioration - escalating oxygen requirements, hypoxia and tachycardia.

> Delays in escalation (nurses to doctors and between doctors).

$>$ Inadequate responses by medical teams to nursing escalations.

$>$ We reviewed escalation and response time to 30 deteriorating patients (new NEWS $\geq 5$ ) and repeated this exercise post intervention.

> We conducted surveys of doctors reviewing perceived level of support when managing deteriorating inpatients. We ran a junior doctors deteriorating patient group and conducted a nursing forum to identify barriers to recognition, escalation and response.

\section{Interventions}

> Multi-faceted education programme

> Tailored teaching sessions for specialties.

> Multidisciplinary deteriorating patient training days with NEWS2 incorporation.

Authors: Homerton University Hospital NHS Foundation Trust ${ }^{*} \mathrm{RCP}$ chief registrar
> In-situ simulation focusing on deterioration.

$>$ Presentation of thematic analysis to hospital-wide meetings.

> Multidisciplinary handovers - Introduction of a night handover checklist. We addressed issues surrounding attendance and integrated nursing presence. We worked with the emergency department to improve the transfer of care.

> Night huddles - New 1am huddles with the hospital at night team to discuss patients, highlight concerns and redistribute workload.

\section{Results and discussion}

Thematic review of ITU admissions

Post-intervention thematic analysis of ITU admissions found a reduction in:

$>$ Delays in referral to ITU ( $31 \%$ to $21 \%$ ).

$>$ Inadequate medical response to deterioration (18\% to $8 \%$ ).

$>$ Delays in nursing response to deterioration (12\% to $4 \%$ ).

We found a reduction in the incidents of staff tolerating prolonged periods of escalating oxygen requirements.

\section{Escalation and response times}

Nursing escalation of deterioration improved with documented evidence in $73.3 \%$ of cases (22/30) compared with $53.3 \%$ (16/30). The medical response times and seniority of reviewer did not change following the interventions.

\section{Conclusion}

Improving response to deterioration can be achieved using a multidisciplinary approach combining education initiatives with systems changes. Obtaining in-depth understanding of local issues around deterioration is best done using qualitative data analysis to support quantitative data such as escalation and response times. This has allowed us to target our interventions and education. Further improvements are required, in particular around medical response times and seniority. To address this we are running a pilot scheme with an additional deteriorating patient doctor on the wards at night. We have developed an escalation and response pathway and critical care referral guidelines. We will review the impact in the next cycle of data collection. 